\title{
Neuropsychiatric Symptoms in Patients with Idiopathic Rapid Eye Movement Sleep Behavior Disorder
}

\author{
Jung-Ick Byun, MD, Yu Yong Shin, MD, Sung-Eun Chung, MD, Won-Chul Shin, MD \\ Department of Neurology, Kyung Hee University Hospital at Gangdong, Seoul, Korea
}

Background and Objective Idiopathic rapid eye movement sleep behavior disorder (IRBD) is a strong predictor of the development of synucleinopathies, including Parkinson's disease. Neuropsychiatric symptoms are common in patients with Parkinson's disease but have not been properly evaluated in IRBD. We used the Symptom Checklist-90-Revision (SCL-90-R) to evaluate the symptom profile of patients with drug-naïve IRBD.

Methods Consecutive drug-naive patients with video-polysomnography-confirmed IRBD that visited Kyung Hee University Hospital at Gangdong sleep clinic January 2009-November 2016 were reviewed. Age- and sex-matched healthy volunteers served as controls. Questionnaires evaluating sleep [Pittsburgh sleep quality index (PSQI) and insomnia severity index (ISI)] and depression [beck depression inventory-II (BDI-II)] were conducted with the SCL-90-R in IRBD patients and controls.

Results Twenty patients with IRBD and 20 age- and sex-matched controls were analyzed. In IRBD patients, the mean age was $59.5,55 \%$ were male, and the mean disease duration was 55.1 months. PSQI scores $(5.7 \pm 2.2$ vs. $3.2 \pm 1.4, \mathrm{p}<0.0001)$ and ISI scores $(11.2 \pm 8.7$ vs. $4.1 \pm 3.4, \mathrm{p}<$ $0.0001)$ were higher in patients with IRBD than in the controls. Three IRBD patients (15\%) had abnormal SCL-90-R T-scores over 70. The T-scores for phobic anxiety $(\mathrm{p}=0.009)$, interpersonal sensitivity $(\mathrm{p}=0.011)$, psychoticism $(\mathrm{p}=0.013)$, hostility $(\mathrm{p}=0.014)$, anxiety $(\mathrm{p}=0.020)$, and depression $(\mathrm{p}=0.049)$ were higher in the patients. After adjusting for age, BDI-II scores were positively correlated with RBD disease duration $(\mathrm{r}=0.484, \mathrm{p}=0.042)$ in IRBD patients.

Conclusions The results of our study demonstrate that neuropsychiatric distress is more severe in patients with IRBD with no comorbidities than in otherwise healthy individuals.

Sleep Med Res 2017;8(2):86-91

Key Words REM sleep behavior disorder, Neuropsychiatric symptom, SCL-90-R.

Received: November 22, 2017

Revised: December 9, 2017

Accepted: December 13, 2017

\section{Correspondence}

Won-Chul Shin, MD

Department of Neurology,

Kyung Hee University Hospital at Gangdong,

892 Dongnam-ro, Gangdong-gu,

Seoul 05278, Korea

Tel +82-2-440-6166

Fax +82-2-440-7242

E-mailsshin1chul@gmail.com

ORCID

Jung-Ick Byun

https://orcid.org/0000-0002-6224-4575

Yu Yong Shin

https://orcid.org/0000-0003-3386-1942

Sung-Eun Chung

https://orcid.org/0000-0001-8334-2249

Won-Chul Shin

https://orcid.org/0000-0003-3044-9397

\section{INTRODUCTION}

Rapid eye movement (REM) sleep behavior disorder (RBD) is a parasomnia that is characterized by the loss of muscle atonia during REM sleep, associated with the complex motor enactment of dreams. ${ }^{1}$ RBD patients free of associated neurologic disorders have idiopathic RBD (IRBD). These patients are at high risk of developing neuro-degenerative diseases, such as Parkinson's disease (PD), multiple system atrophy, and dementia with Lewy bodies..$^{2-4}$ Longitudinal studies showed that $52-82 \%$ of IRBD patients acquire a neuro-degenerative disorder within 12 years of disease onset. ${ }^{3,5}$ Early non-motor features of Parkinsonism, such as impaired color vision, olfaction, and autonomic function, are observed in IRBD. ${ }^{6}$ IRBD is therefore widely viewed as a prodromal stage of synucleinopathies.

Neuropsychiatric symptoms, including depression, anxiety and apathy, are frequently reported in PD. ${ }^{7}$ Depression affects up to $35 \%{ }^{8}$ and anxiety occurs in $40 \%$ of the patients with PD. ${ }^{9}$ Even drug-naive patients in the early stages of the disease stage exhibit more symptoms of depression and anxiety than are observed in controls. ${ }^{10}$ The results of a questionnaire study [Symptom 
Checklist 90-Revised (SCL-90-R)] showed that half of the patients with PD exhibited somatization, depression, anxiety, or obsessive-compulsive behaviors. ${ }^{11}$ Assessing neuropsychiatric symptoms is critical in PD because they can adversely affect the course of disease and the well-being of the patient ${ }^{12}$ and increase mortality. ${ }^{13}$ Those with major depression experience faster cognitive and functional decline and display an increased need for antiParkinsonian therapy than are observed in those without these symptoms. ${ }^{14}$

Neuropsychiatric symptoms are often unrecognized and therefore untreated in patients with IRBD. Most studies evaluate PD patients with comorbid RBD, that negatively effects non-motor symptoms in these patients. They display more symptoms of depression, sleepiness, and cognitive impairment than are observed in similar patients without RBD. ${ }^{15}$ Additionally, PD patients with RBD report more anxiety at baseline and that this anxiety increases over time. ${ }^{16}$ Only a few studies have evaluated IRBD patients. One study compared personality changes between PD and RBD patients and showed similar pattern of personality changes compared to a control population more neurotic, less extraverted and less open. ${ }^{17}$ Another study showed that depression was more frequent in IRBD. ${ }^{18}$ Assessing neuropsychiatric symptoms, such as depression, in patients with IRBD is therefore crucial because depression is independently associated with poor quality of life. ${ }^{19}$

We hypothesized that IRBD patients have more severe neuropsychiatric disturbance than controls, which is associated with neurodegenerative process. SCL-90-R is a structured self-reported questionnaire that is used to evaluate psychopathology and psychological distress. We used the SCL-90-R to evaluate the prevalence of neuropsychiatric symptoms and psychological distress in IRBD patients and compared the results to those obtained in age- and sex-matched controls.

\section{METHODS}

\section{Participants}

Consecutive drug-naïve patients with video polysomnography (PSG)-confirmed IRBD that visited Kyung Hee University Hospital at Gangdong sleep clinic January 2009-November 2016 were reviewed. RBD was diagnosed according to the International Classification of Sleep Disorders (ICSD-3) criteria. ${ }^{1}$ Those with 1) a neuro-degenerative disease, other neurological, or psychiatric disorder; 2) severe medical illness; 3) moderate to severe obstructive sleep apnea (OSA) (apnea-hypopnea index $\geq 15 / \mathrm{hr}$ ), or 4) other sleep disorders [including periodic limb movement disorder (PLMD)] were excluded. Age- and sex-matched healthy volunteers from the same region as the IRBD patients served as controls. The controls were screened for sleep-related symptoms and neurological or psychological diseases using a structured questionnaire in addition to a clinical interview.
This study was approved by the Institutional Review Board of the Kyung Hee University Hospital at Gangdong (IRB No: 201409023-001). Written informed consent to participate was obtained from all enrolled patients.

\section{Procedures}

Questionnaires evaluating sleep [Pittsburgh sleep quality in$\operatorname{dex}$ (PSQI); ${ }^{20}$ Epworth sleepiness scale (ESS); ${ }^{21}$ Sanford sleepiness scale (SSS); ${ }^{22}$ and insomnia severity index (ISI) $\left.{ }^{23}\right]$ and depression [beck depression inventory-II (BDI-II) ${ }^{24}$ ] in addition to the SCL-90-R were completed by IRBD patients and controls.

The SCL-90-R is composed of 90 questions, each of which is scored on a 5-point scale ranging from "not at all" = 0 to "extremely often" $=5$. The patient's symptoms are assigned to the following 9 categories: somatization (SOM), obsession-compulsion (O-C), interpersonal sensitivity (I-S), depression (DEP), anxiety (ANX), hostility (HOS), phobic anxiety (PHOB), paranoid ideation (PAR), and psychoticism (PSY). Additionally, the following three global indices are used to measure and calculate overall psychological distress: the global severity index (GSI) and the positive symptom total (PST) index, and positive symptom distress index (PSDI). The standard Korean version of the SCL-90-R ${ }^{25}$ was used, and the result of the questionnaire was presented as a $\mathrm{T}$ score with a mean of 50 and a standard deviation of 10. A score of 70 (two standard deviations from the mean) was considered to indicate clinically abnormal. ${ }^{26}$

\section{Statistical Analysis}

All data are presented as the mean \pm SD. The Mann-Whitney U-test was used to evaluate group differences in demographics and questionnaire scores. We ranked the patients according to the proportion of positive SCL-90-R items (scores above 1). We listed the 10 most common items found in the IRBD groups and used Fisher's exact test to compare the frequency of each item with that observed in the controls. The correlations between SCL-90-R T-scores and clinical variables were tested using Spearman's correlation after adjusting for age. The significance level was set to 0.05 . All statistical comparisons were conducted using SPSS (Version 22.0, IBM Corp., Armonk, NY, USA).

\section{RESULTS}

\section{Patient Characteristics}

Initially, 38 patients with IRBD were enrolled. Three patients were excluded for moderate or severe OSA, and 15 were excluded for concomitant PLMD. Eventually, 20 patients with IRBD and 20 age- and sex-matched controls were analyzed. The mean age of the IRBD patients was 58, and half of them were male. The mean disease duration was 55.1 months, and the mean symptom frequency was 3.7 days per week. PSQI scores were overall higher in patients with IRBD than in the controls $(5.7 \pm 2.2$ vs. 
$3.2 \pm 1.4, \mathrm{p}<0.0001)$ and specifically higher in the following components: subjective sleep quality $(\mathrm{p}<0.0001)$, sleep latency $(\mathrm{p}=0.012)$, sleep duration $(\mathrm{p}=0.004)$, and sleep disturbance $(\mathrm{p}$

Table 1. Demographics, sleep questionnaire scores, and PSG results

\begin{tabular}{|c|c|c|c|}
\hline Characteristic & $\begin{array}{l}\text { Control } \\
(\mathrm{n}=20)\end{array}$ & $\begin{array}{c}\text { IRBD } \\
(\mathrm{n}=20)\end{array}$ & p-value* \\
\hline Age (year) & $59.5 \pm 5.0$ & $58.5 \pm 8.0$ & 0.968 \\
\hline Sex (male, \%) & $9(45.0)$ & $11(55.0)$ & 0.752 \\
\hline BMI $\left(\mathrm{kg} / \mathrm{m}^{2}\right)$ & $23.5 \pm 1.7$ & $23.3 \pm 3.1$ & 0.499 \\
\hline Disease duration (months) & na & $55.1 \pm 54.9$ & \\
\hline Symptom frequency (/week) & na & $3.7 \pm 2.3$ & \\
\hline \multicolumn{4}{|l|}{ Questionnaire } \\
\hline PSQI total & $3.2 \pm 1.4$ & $5.7 \pm 2.2$ & $<0.0001$ \\
\hline $\begin{array}{l}\text { C1 (subjective sleep } \\
\text { quality) }\end{array}$ & $0.7 \pm 0.5$ & $1.5 \pm 0.6$ & $<0.0001$ \\
\hline C2 (sleep latency) & $0.7 \pm 0.6$ & $1.3 \pm 0.9$ & 0.012 \\
\hline C3 (sleep duration) & $0.1 \pm 0.3$ & $0.8 \pm 0.9$ & 0.004 \\
\hline $\begin{array}{l}\text { C4 (habitual sleep } \\
\text { efficiency) }\end{array}$ & 0 & 0 & 1 \\
\hline C5 (sleep disturbance) & $1.1 \pm 0.4$ & $1.4 \pm 0.5$ & 0.022 \\
\hline $\begin{array}{l}\text { C6 (use of sleep } \\
\text { medication) }\end{array}$ & $0.3 \pm 0.7$ & $0.2 \pm 0.7$ & 0.655 \\
\hline C7 (daytime dysfunction) & $0.5 \pm 0.5$ & $0.6 \pm 0.7$ & 0.781 \\
\hline ESS & $4.6 \pm 1.5$ & $4.4 \pm 2.8$ & 0.779 \\
\hline SSS & $2.2 \pm 0.6$ & $2.3 \pm 0.8$ & 0.651 \\
\hline ISI & $4.1 \pm 3.4$ & $9.7 \pm 0.6$ & $<0.0001$ \\
\hline BDI-II & $10.5 \pm 5.1$ & $11.2 \pm 8.7$ & 0.473 \\
\hline \multicolumn{4}{|l|}{ PSG } \\
\hline TST (min) & na & $302.4 \pm 34.5$ & \\
\hline N1 (\%) & na & $19.4 \pm 9.5$ & \\
\hline N2 (\%) & na & $33.5 \pm 11.8$ & \\
\hline N3 (\%) & na & $31.0 \pm 10.1$ & \\
\hline REM (\%) & na & $16.1 \pm 6.6$ & \\
\hline $\mathrm{SL}(\mathrm{min})$ & na & $15.6 \pm 20.1$ & \\
\hline SE (\%) & na & $81.3 \pm 9.8$ & \\
\hline $\mathrm{AI}(/ \mathrm{hr})$ & na & $26.0 \pm 10.3$ & \\
\hline $\mathrm{AHI}(/ \mathrm{hr})$ & na & $2.8 \pm 3.7$ & \\
\hline RDI (/hr) & na & $7.4 \pm 7.9$ & \\
\hline PLMAI (/hr) & na & $0.3 \pm 0.4$ & \\
\hline PLMI (/hr) & na & $2.6 \pm 5.8$ & \\
\hline
\end{tabular}

Data are expressed as the mean \pm standard deviation.

*Mann-Whitney U test.

IRBD: idiopathic rapid eye movement sleep behavior disorder; BMI: body-mass index; PSQI: Pittsburgh sleep quality index, ESS: Epworth sleepiness scale, SSS: Sanford sleepiness scale, ISI: insomnia severity index, BDI-II: beck depression inventory-II, PSG: polysomnography, REM: rapid eye movement, TST: total sleep time, SL: sleep latency, SE: sleep efficacy, AI: rousal index, AHI: apneahypopnea index, RDI: respiratory distress index, PLMAI: periodic limb movement arousal index, PLMI: periodic limb movement index, na: not available.
$=0.022)$. ISI was also higher in IRBD $(9.7 \pm 0.6$ vs. $4.1 \pm 3.4$, $\mathrm{p}$ $<0.0001)$. The results of other sleep questionnaires and BDI-II scores were similar between the two groups. The results of the PSG data in the IRBD patients is described in Table 1.

\section{SCL-90-R}

Except for SOM, O-C, and PAR, SCL-90-R T-scores were significantly higher in IRBD patients than in the controls, as follows: PHOB ( $p=0.009)$, I-S ( $p=0.011)$, PSY ( $p=0.013)$, HOS $(\mathrm{p}=0.014), \operatorname{ANX}(\mathrm{p}=0.020)$, and DEP $(\mathrm{p}=0.049)$ (Fig. 1). Three IRBD patients (15\%) had abnormal T-scores over 70 in one or more subscale (one patients in I-S, PHOB, PAR, and PSY; one in O-C, I-S, DEP, ANX, HOST, PAR, and PSY; and one in SOM).

In IRBD patients, the most common positive item in the SCL90-R test was "Loss of sexual interest or pleasure." However, the proportion was like that observed in the controls. Among the 10 most-positive items in the IRBD patients, "Trouble remembering things," a subscale of $\mathrm{O}-\mathrm{C}$, and "Feeling very self-conscious with others", a subscale of I-S, were more common in IRBD patients than in the controls (Table 2).

After adjusting for age, BDI-II was positively correlated with $\mathrm{RBD}$ disease duration $(\mathrm{r}=0.484, \mathrm{p}=0.042)$. There was no significant correlation between SCL-90-R T-scores and RBD disease duration or symptom frequency. Only PST $(r=0.465, \mathrm{p}=0.052)$, GSI ( $\mathrm{r}-0.443, \mathrm{p}=0.065)$, and HOS $(\mathrm{r}=0.448, \mathrm{p}=0.063)$ tended to be positively correlated with RBD duration. PSQI score were significantly correlated with $\mathrm{PHOB}(\mathrm{r}=0.49, \mathrm{p}=0.039)$ and PSY $(\mathrm{r}=0.532, \mathrm{p}=0.023)$ in RBD patients.

\section{DISCUSSION}

We conducted systematic neuropsychologic evaluations using the SCL-90-R in IRBD patients and age- and sex-matched healthy controls. Even after we excluded individuals with comorbid neurologic, psychiatric, or sleep disorders, neuropsychiatric distress in the I-S, PHOB, PSY, HOS, ANX, and DEP domains was more severe in the patients with IRBD. Three of the patients (15\%) had an abnormal psychological profile with SCL90-R T-scores higher than 70.

In IRBD, sleep disturbance can cause psychological distress. Disturbed sleep has been associated with psychiatric symptoms. ${ }^{27,28}$ The IRBD patients had higher PSQI scores than were observed in the controls even after we excluded individuals with other sleep disorders, such as periodic limb movement during sleep, or moderate to severe OSA. PSQI scores were significantly correlated with T-scores for the PHOB and PSY subscales. The PSQI component encompassing sleep disturbance and sleep medication has been higher in IRBD. ${ }^{29}$ Our patients scored higher in sleep disturbance components as well as in sleep latency, sleep duration and sleep quality components. The use of sleep medication was similar in our patients because we includ- 


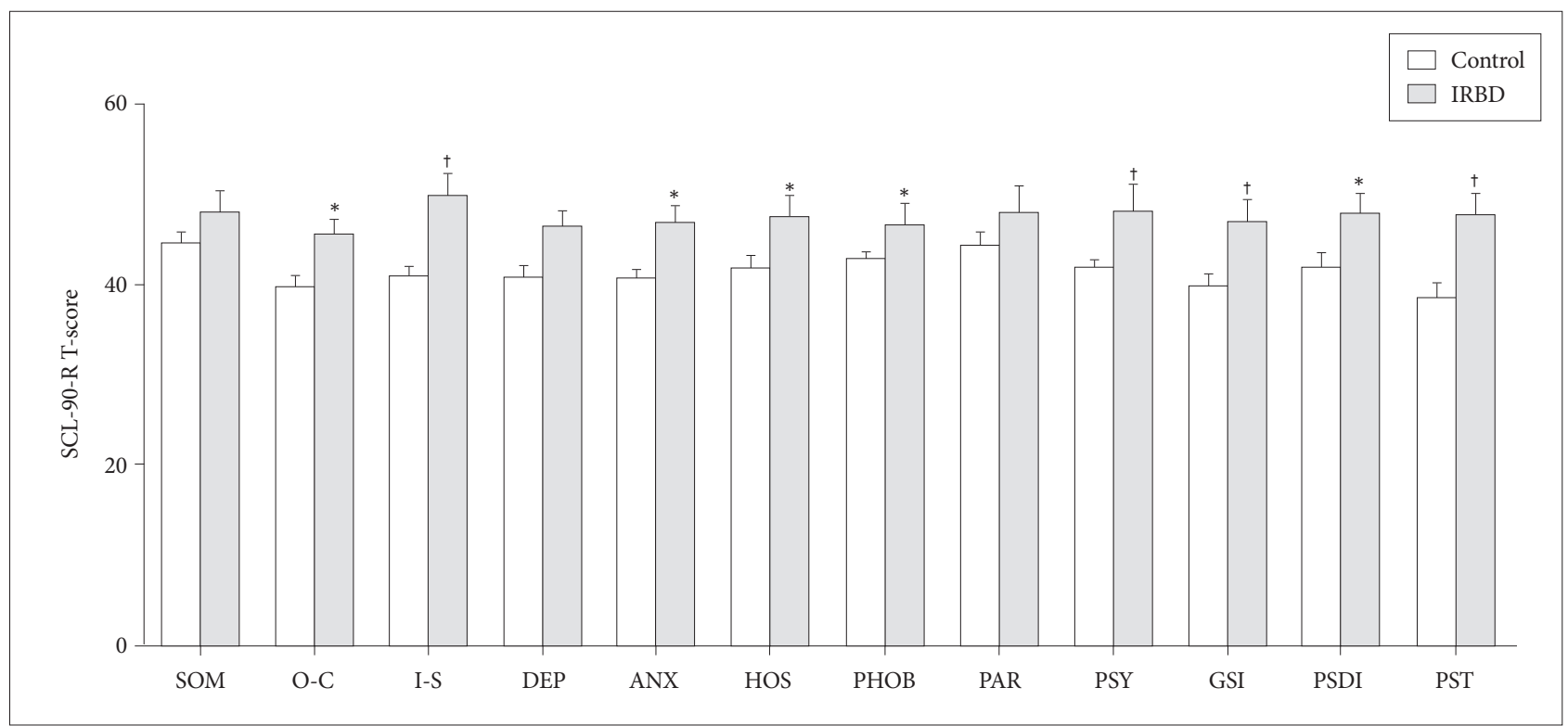

Fig. 1. SCL-90-R T-scores in IRBD patients and controls. ${ }^{*} p<0.05$ compared with the control, ${ }^{\dagger} p<0.01$ compared with the control; MannWhitney U test. SCL-90-R: Symptom Checklist-90-Revised, IRBD: idiopathic rapid eye movement sleep behavior disorder, SOM: somatization, O-C: obsession-compulsion, I-S: interpersonal sensitivity, DEP: depression, ANX: anxiety, HOS: hostility, PHOB: phobic anxiety, PAR: paranoid ideation, PSY: psychoticism, GSI: global severity index, PSDI: positive symptom distress index, PST: positive symptom total.

Table 2. Comparison of the 10 most common positive items

\begin{tabular}{rlccc}
\hline & 10 most common positive items in SCL-90-R & IRBD (n=20) & Control (n=20) & p-value \\
\hline 5 & Loss of sexual interest or pleasure & $11(55.0)$ & $9(35.0)$ & 0.341 \\
9 & Trouble remembering things & $9(45.0)$ & $1(5.0)$ & 0.008 \\
69 & Feeling very self-conscious with others & $7(40.0)$ & $1(5.0)$ & 0.020 \\
27 & Pains in lower back & $6(30.0)$ & $1(5.0)$ & 1 \\
45 & Having to check and double-check what you do & $6(30.0)$ & $1(5.0)$ & 0.091 \\
68 & Having ideas or beliefs that others do not share & $7(35.0)$ & $4(20.0)$ & 0.091 \\
79 & Feelings of worthlessness & $5(25.0)$ & $2(10.0)$ & 0.480 \\
29 & Feeling lonely & $5(25.0)$ & $2(10.0)$ & 0.407 \\
44 & Trouble falling asleep & $4(20.0)$ & 0 & 0.407 \\
26 & Blaming yourself for things & & 0.106 \\
\hline
\end{tabular}

Number of patients (percentage), p-value for Fisher's exact test.

ed only drug-naïve IRBD patients. Because we excluded controls with subjective sleep disturbances, our PSQI score results may be biased. However, all of the patients with IRBD had a PSQI score above 5, and this is a significantly higher proportion than was achieved in a previous report (68.7\%). ${ }^{29}$ The absence of sleep medication, such as clonazepam may have resulted higher rates of sleep disturbance in our patients than the previous reported results. The sleep fragmentation observed in IRBD may therefore be caused by unstable wake/sleep and REM/NREM transitions, ${ }^{30}$ in line with the findings of a previous study. ${ }^{29}$

Phobic anxiety was the most prominent neuropsychiatric symptom observed in IRBD, followed by interpersonal sensitivity. Patients with IRBD often experience unpleasant dreams, and this can sometimes result in "sleep phobia", that can increase phobic anxiety in affected patients. Some of these patients en- gage in sleep talking, shouting, screaming, hitting, or punching. Abnormal behavior and vocalization during sleep can cause embarrassment when sleeping with partners or guests or in hotels. ${ }^{31}$ Such behavior can increase self-consciousness and negative expectations about interpersonal reactions. A previous study showed that RBD patients experience more aggressive episodes in their dreams but exhibit normal daytime aggressiveness. ${ }^{32}$ However, the IRBD patients in our study displayed more severe hostility during the daytime than the controls. Compared with a previous study that included predominantly male patients and controls, about half of the patients in this study were female. Although males are typically more likely to show aggression, ${ }^{33}$ hostility related to IRBD may be more significant or obvious in female patients. 
The neuropsychologic disturbances observed in IRBD may partially be caused by neuro-degenerative processes. A large prospective study showed that in otherwise healthy men, phobic anxiety is a risk factor for PD. ${ }^{34}$ The structures that are involved in anxiety or depression, such as the raphe nucleus and locus coeruleus, are commonly affected by Lewy bodies in patients with PD or RBD. ${ }^{31}$ Decreased striatal dopamine transporter availability has been associated with symptoms of depression and anxiety in PD patients $s^{35,36}$ and has been identified in IRBD patients. ${ }^{37}$ Although BDI-II score were similar between IRBD and the controls, it was correlated with the RBD disease duration after adjusting for age, suggesting an association between disease progression and the severity of depressive symptoms. An increase in psychoticism may be associated with neuro-degenerative processes in dementia with Lewy body disease, a key feature of which is visual hallucinations. Lewy body pathology in the projections of the substantia nigra pars reticulata to brainstem nuclei can result in the activation of limbic structures, thereby causing hallucinations.

This study is the first to systematically evaluate the neuropsychiatric symptoms in IRBD patients without comorbid conditions. However, any interpretation of this study should be made in consideration of its limitations. Because this study used a cross-sectional design, it is difficult to evaluate any causal relationship between IRBD and neuropsychiatric distress. The number of patients was small, and therefore we were not able to adjust possible confounders that can affect SCL-90-R score, such as PSQI or ISI. Moreover, there is a risk for false-positive findings resulting from multiple testing. However, we strictly limited inclusion to drug-naïv IRBD patients without comorbid conditions so that we could better evaluate the true effect of RBD on neuropsychiatric symptoms. Finally, PSG was conducted only in the IRBD patients, and controls with sleep disturbances were excluded.

In this study, we found that the IRBD patients exhibited more psychosomatic distress than was observed in the controls and $15 \%$ of the IRBD patients suffered abnormal neuropsychiatric symptoms. These symptoms should not be overlooked because they can decrease the patient's quality of life and potentially adversely affect the course of neuro-degeneration. The results of our study support the notion that RBD and neuropsychiatric symptoms occur before the onset of neurodegenerative disease. The different neuropsychiatric symptoms observed in IRBD may predict different types of synucleinopathies, such as PD or dementia with Lewy bodies. Further prospective studies that include larger numbers of patients should be conducted to explore this issue.

\section{Acknowledgments}

This study was supported by the Basic Science Research Program of the National Research Foundation of Korea (NRF) (No. NRF-2016R1D1A1B0 3934722).

\section{Conflicts of Interest}

The authors have no financial conflicts of interest.

\section{Authors' Contribution}

Conceptualization: Byun JI. Data curation: Shin YY, Chung SE. Formal analysis: Byun JI. Investigation: Shin YY, Chung SE. Writing-original draft: Byun JI. Writing—review \& editing: Byun JI, Shin WC. Supervison: Shin WC.

\section{REFERENCES}

1. American Academy of Sleep Medicine. International Classification of Sleep Disorders-Third Edition (ICSD-3). Darien: American Academy of Sleep Medicine 2014.

2. Schenck CH, Bundlie SR, Mahowald MW. Delayed emergence of a parkinsonian disorder in $38 \%$ of 29 older men initially diagnosed with idiopathic rapid eye movement sleep behaviour disorder. Neurology 1996;46:388-93

3. Postuma RB, Gagnon JF, Vendette M, Fantini ML, Massicotte-Marquez J, Montplaisir J. Quantifying the risk of neurodegenerative disease in idiopathic REM sleep behavior disorder. Neurology 2009;72:1296300.

4. Iranzo A, Molinuevo JL, Santamaría J, Serradell M, Martí MJ, Valldeoriola F, et al. Rapid-eye-movement sleep behaviour disorder as an early marker for a neurodegenerative disorder: a descriptive study. Lancet Neurol 2006;5:572-7.

5. Iranzo A, Tolosa E, Gelpi E, Molinuevo JL, Valldeoriola F, Serradell M, et al. Neurodegenerative disease status and post-mortem pathology in idiopathic rapid-eye-movement sleep behaviour disorder: an observational cohort study. Lancet Neurol 2013;12:443-53.

6. Postuma RB, Lang AE, Massicotte-Marquez J, Montplaisir J. Potential early markers of Parkinson disease in idiopathic REM sleep behavior disorder. Neurology 2006;66:845-51.

7. Schapira AHV, Chaudhuri KR, Jenner P. Non-motor features of Parkinson disease. Nat Rev Neurosci 2017;18:509.

8. Reijnders JS, Ehrt U, Weber WE, Aarsland D, Leentjens AF. A systematic review of prevalence studies of depression in Parkinson's disease. Mov Disord 2008;23:183-9; quiz 313.

9. Walsh K, Bennett G. Parkinson's disease and anxiety. Postgrad Med J 2001;77:89-93.

10. Aarsland D, Brønnick K, Alves G, Tysnes OB, Pedersen KF, Ehrt U, et al. The spectrum of neuropsychiatric symptoms in patients with early untreated Parkinson's disease. J Neurol Neurosurg Psychiatry 2009;80: 928-30.

11. Bugalho P, da Silva JA, Cargaleiro I, Serra M, Neto B. Psychiatric symptoms screening in the early stages of Parkinson's disease. J Neurol 2012;259:124-31.

12. Weintraub D, Stern MB. Psychiatric complications in Parkinson disease. Am J Geriatr Psychiatry 2005;13:844-51.

13. Pachana NA, Egan SJ, Laidlaw K, Dissanayaka N, Byrne GJ, Brockman $\mathrm{S}$, et al. Clinical issues in the treatment of anxiety and depression in older adults with Parkinson's disease. Mov Disord 2013;28:1930-4.

14. Ravina B, Camicioli R, Como PG, Marsh L, Jankovic J, Weintraub D, et al. The impact of depressive symptoms in early Parkinson disease. Neurology 2007;69:342-7.

15. Rolinski M, Szewczyk-Krolikowski K, Tomlinson PR, Nithi K, Talbot $\mathrm{K}$, Ben-Shlomo Y, et al. REM sleep behaviour disorder is associated with worse quality of life and other non-motor features in early Parkinson's disease. J Neurol Neurosurg Psychiatry 2014;85:560-6.

16. Rutten S, van der Ven PM, Weintraub D, Pontone GM, Leentjens AFG, Berendse HW, et al. Predictors of anxiety in early-stage Parkinson's disease-Results from the first two years of a prospective cohort study. Parkinsonism Relat Disord 2017;43:49-55.

17. Baig F, Lawton MA, Rolinski M, Ruffmann C, Klein JC, Nithi K, et al. Personality and addictive behaviours in early Parkinson's disease and 
REM sleep behaviour disorder. Parkinsonism Relat Disord 2017;37:72-8.

18. Vilas D, Iranzo A, Pont-Sunyer C, Serradell M, Gaig C, Santamaria J, et al. Brainstem raphe and substantia nigra echogenicity in idiopathic REM sleep behavior disorder with comorbid depression. J Neurol 2015;262:1665-72.

19. Kim KT, Motamedi GK, Cho YW. Quality of life in patients with an idiopathic rapid eye movement sleep behaviour disorder in Korea. $J$ Sleep Res 2017;26:422-7.

20. Sohn SI, Kim DH, Lee MY, Cho YW. The reliability and validity of the Korean version of the Pittsburgh Sleep Quality Index. Sleep Breath 2012;16:803-12.

21. Cho YW, Lee JH, Son HK, Lee SH, Shin C, Johns MW. The reliability and validity of the Korean version of the Epworth sleepiness scale. Sleep Breath 2011;15:377-84.

22. Hoddes E, Zarcone V, Smythe H, Phillips R, Dement WC. Quantification of sleepiness: a new approach. Psychophysiology 1973;10:431-6.

23. Cho YW, Song ML, Morin CM. Validation of a Korean version of the insomnia severity index. J Clin Neurol 2014;10:210-5.

24. Jo SA, Park MH, Jo I, Ryu SH, Han C. Usefulness of Beck Depression Inventory (BDI) in the Korean elderly population. Int J Geriatr Psychiatry 2007;22:218-23.

25. Kim JH, Kim KI. The standardization study of symptom checklist-90revision in Korea III. Ment Health Res 1984;2:278-311.

26. Derogatis LR. SCL-90-R: administration, scoring and procedures manual. Minneapolis: National Computer Systems Inc. 1994.

27. Berlin RM, Litovitz GL, Diaz MA, Ahmed SW. Sleep disorders on a psychiatric consultation service. Am J Psychiatry 1984;141:582-4.

28. Ford DE, Kamerow DB. Epidemiologic study of sleep disturbances and psychiatric disorders. An opportunity for prevention? JAMA 1989;262: 1479-84.

29. Postuma RB, Gagnon JF, Pelletier A, Montplaisir JY. Insomnia and somnolence in idiopathic RBD: a prospective cohort study. NPJ Parkinsons Dis 2017;3:9.

30. Christensen JAE, Jennum P, Koch H, Frandsen R, Zoetmulder M, Arvastson L, et al. Sleep stability and transitions in patients with idiopathic REM sleep behavior disorder and patients with Parkinson's disease. Clin Neurophysiol 2016;127:537-43.

31. Boeve BF. REM sleep behavior disorder: updated review of the core features, the REM sleep behavior disorder-neurodegenerative disease association, evolving concepts, controversies, and future directions. Ann NY Acad Sci 2010;1184:15-54.

32. Fantini ML, Corona A, Clerici S, Ferini-Strambi L. Aggressive dream content without daytime aggressiveness in REM sleep behavior disorder. Neurology 2005;65:1010-5.

33. Bettencourt BA, Miller N. Gender differences in aggression as a function of provocation: a meta-analysis. Psychol Bull 1996;119:422-47.

34. Weisskopf MG, Chen H, Schwarzschild MA, Kawachi I, Ascherio A. Prospective study of phobic anxiety and risk of Parkinson's disease. Mov Disord 2003;18:646-51.

35. Weintraub D, Newberg AB, Cary MS, Siderowf AD, Moberg PJ, Kleiner-Fisman G, et al. Striatal dopamine transporter imaging correlates with anxiety and depression symptoms in Parkinson's disease. J Nucl Med 2005;46:227-32.

36. Remy P, Doder M, Lees A, Turjanski N, Brooks D. Depression in Parkinson's disease: loss of dopamine and noradrenaline innervation in the limbic system. Brain 2005;128:1314-22.

37. Iranzo A, Lomeña F, Stockner H, Valldeoriola F, Vilaseca I, Salamero M, et al. Decreased striatal dopamine transporter uptake and substantia nigra hyperechogenicity as risk markers of synucleinopathy in patients with idiopathic rapid-eye-movement sleep behaviour disorder: a prospective study [corrected]. Lancet Neurol 2010;9:1070-7. 